\title{
REDUCED BRANCHING PROCESSES WITH VERY HEAVY TAILS
}

\author{
ANDREAS N. LAGERÅS, *** Chalmers University of Technology and Göteborg University \\ SERIK SAGITOV, ${ }^{*}$ Chalmers University of Technology
}

\begin{abstract}
The reduced Markov branching process is a stochastic model for the genealogy of an unstructured biological population. Its limit behavior in the critical case is well studied for the Zolotarev-Slack regularity parameter $\alpha \in(0,1]$. We turn to the case of very heavytailed reproduction distribution $\alpha=0$ assuming that Zubkov's regularity condition holds with parameter $\beta \in(0, \infty)$. Our main result gives a new asymptotic pattern for the reduced branching process conditioned on nonextinction during a long time interval.
\end{abstract}

Keywords: Reduced branching process; critical branching process; heavy tail; regular variation

2000 Mathematics Subject Classification: Primary 60J80

Secondary 60F05

\section{Introduction}

A single type branching process describes a population of particles with independent and identical reproduction laws. In the Markov branching process with continuous time each particle lives an exponential time with mean 1 and at the moment of death splits into a random number of daughter particles. If we assume that the branching system starts at time 0 from a single particle with $v$ daughters then the whole process is defined by the distribution of the random variable $v$. In the critical case when the average number of daughters is exactly $1, \mathrm{E}[v]=1$, the limit behavior of the branching process is studied under the following Zolotarev-Slack regularity condition (cf. [8] and [10]). The generating function $f(s)=\mathrm{E}\left[s^{\nu}\right]$ is assumed to satisfy

$$
f(s)=s+(1-s)^{1+\alpha} L\left(\frac{1}{1-s}\right), \quad 0 \leq \alpha \leq 1,
$$

where $L$ is slowly varying at $\infty$. When the variance of $v$ is finite, the condition holds with $\alpha=1$ and $L$ tends to a constant, while the case in which $0<\alpha<1$ is usually referred to as the infinite-variance case and is well studied in the literature.

We turn to the less studied case in which $\alpha=0$, focussing on a special class of slowly varying functions (which was initially introduced in [11]):

$$
L(x) \sim(\ln x)^{-\beta} L_{1}(\ln x), \quad \beta>0, x \rightarrow \infty,
$$

where $L_{1}$ is another slowly varying function. Given (1) and $\alpha=0$, Zubkov's regularity condition (2) is equivalent to the following requirement on the tail distribution function (see Lemma 1, below):

$$
\mathrm{P}(v>k) \sim \beta k^{-1}(\ln k)^{-1-\beta} L_{1}(\ln k), \quad k \rightarrow \infty .
$$

Received 16 October 2007; revision received 16 January 2008.

* Postal address: Mathematical Sciences, Chalmers University of Technology, 41296 Göteborg, Sweden.

** Email address: andreas@math.su.se 
Note that in this case $\mathrm{E}\left[v\left(\ln _{+} v\right)^{\beta-\varepsilon}\right]<\infty$ and $\mathrm{E}\left[v\left(\ln _{+} v\right)^{\beta+\varepsilon}\right]=\infty$ for all $\varepsilon>0$. This is a consequence of [1, Theorem 8.1.8].

The main characteristic of the branching process is the number of particles $Z(t)$ alive at time $t$. The key issues of the asymptotics of the nonextinction probability $Q(t)=\mathrm{P}(Z(t)>0)$ as $t \rightarrow \infty$ and the limit behavior of $Z(t)$ conditioned on nonextinction in the case in which $\alpha=0$ were recently addressed by Nagaev and Wachtel [4]. They considered the discrete-time version of the Markov branching process and obtained general limit results without the extra assumption (2). In Section 4 we give a direct proof (which is more straightforward than the counterpart of the Nagaev-Wachtel proof) of the following result with the extra condition.

Theorem 1. If (1) holds with $\alpha=0$ and L satisfies (2) then

$$
Q(t)=\exp \left(-t^{1 /(1+\beta)} L_{q}(t)\right),
$$

where $L_{q}(t)$ is such a slowly varying function as $t$ tends to $\infty$ that

$$
L_{q}^{1+\beta}(t) \sim(1+\beta) L_{1}\left(t^{1 /(1+\beta)} L_{q}(t)\right) .
$$

Furthermore, there exists a regularly varying function (see (29), below)

$$
c(t)=t^{\beta(1+\beta)^{-2}} L_{c}(t)
$$

such that, for all $x \geq 0$,

$$
\mathrm{P}\left(Z(t) \leq \mathrm{e}^{x c(t)} \mid Z(t)>0\right) \rightarrow 1-\exp \left(-x^{\beta+1}\right), \quad t \rightarrow \infty .
$$

There is a striking feature in the asymptotics of $Z(t)$ which was pointed out to us by V. Wachtel. Note that the regular variation index $\beta(1+\beta)^{-2}$ of the scaling function $c(t)$ increases as $\beta$ goes from $\infty$ down to $\beta=1$. As the reproduction tail becomes heavier, this is what we expect to happen, namely, we expect to have a larger asymptotic value for the population size $Z(t)$ at survival. What is puzzling about (6), however, is that as $\beta$ falls below the threshold value 1 , the corresponding scaling function $c(t)$ attributes a smaller size for the surviving population despite the fact that the reproduction tail becomes even heavier.

Some light on this phenomenon is shed by the following seminal results of [11]. If $\tau(t)$ is the time to the most recent common ancestor for all particles alive at time $t$ then, under condition (1), with $0<\alpha \leq 1$,

$$
\mathrm{P}(\tau(t) \leq t x \mid Z(t)>0) \rightarrow x, \quad t \rightarrow \infty,
$$

while, under the conditions of Theorem 1 ,

$$
\mathrm{P}(\tau(t) \leq t x \mid Z(t)>0) \rightarrow x^{\beta /(1+\beta)}, \quad t \rightarrow \infty .
$$

The latter means that the ratio $\tau(t) / t$ is asymptotically distributed over $[0,1]$ with the density function

$$
\phi_{\beta}(x)=\beta(1+\beta)^{-1} x^{-1 /(1+\beta)} .
$$

That is, if $\beta$ is changed towards smaller values then the time to the most recent common ancestor will become shorter, so that the growth of the population takes place during a shorter time span. All other things equal, we would expect a decrease in the population size. This effect thus counteracts the increase in size due to heavier tails and, for low enough values of $\beta$, the size of 
the surviving population actually decreases with decreasing $\beta$. Why the threshold value should be $\beta=1$ is an interesting open problem.

In Section 2 we present the so-called reduced branching process describing the genealogy of the particles alive at time $t$. In this section we recall the known limit processes for the reduced branching processes in the cases in which $0<\alpha \leq 1$ obtained in [3] and [9]. Then we state the main result of this paper, Theorem 2, giving a new limit structure as $t$ tends to $\infty$ for the reduced branching process in the case in which $\alpha=0$ and (2) holds. Our Theorem 2 is an extension of (8) in the same manner as the results by [3] and [9] are extensions of (7). It is worth mentioning that in Theorem 2 we do not loose much generality by assuming that (2) holds in the case in which $\alpha=0$ since, by Zubkov's proof, it actually follows that nondegenerate limit distributions of $\tau(t) / t$ must be either of the form (7) or (8) for critical branching processes. In Section 3 we establish some preliminary results, and in Section 4 we prove Theorems 1 and 2.

\section{Limit theorem for the reduced branching process}

Let $Z(u, t)$ stand for the number of particles at time $u$ which stay alive or have descendants at a later time $t \geq u$. For a given time horizon $t$, the process $\{Z(u, t), 0 \leq u \leq t\}$ is called the reduced branching process. The term reduced reflects the fact that we count only those branches in the full genealogical tree that reach to the time of observation $t$. Clearly,

$$
\mathrm{P}(Z(u, t)=1)=\mathrm{P}(\tau(t) \leq t-u),
$$

where $\tau(t)$ is the time to the most recent common ancestor. If (1) holds with $0<\alpha \leq 1$ then the limit theorem (7) for the time to the most recent ancestor is extended to the following limit theorem for the reduced branching process by the results of [3] and [9]:

$$
(Z(t x, t) \mid Z(t)>0) \stackrel{\mathrm{D}}{\rightarrow} Z_{\alpha}(-\log (1-x)),
$$

where the convergence of the processes over the time interval $0 \leq x<1$ holds in the Skorohod sense.

Here the limit is a time-transformed Markov branching process $Z_{\alpha}(t)$, whose generating functions can be written down explicitly. The particles of this process live exponential times with mean 1 and at the moment of death produce offspring with the generating function

$$
f_{\alpha}(s)=s+\frac{1}{\alpha}(1-s)\left((1-s)^{\alpha}-1\right) .
$$

This process is supercritical with the offspring number $v_{\alpha}$ having mean $f_{\alpha}^{\prime}(1)=1+1 / \alpha$ and a distribution over $k=2,3, \ldots$ with probabilities

$$
\mathrm{P}\left(v_{\alpha}=k\right)= \begin{cases}\mathbf{1}_{\{k=2\}} & \text { if } \alpha=1, \\ \frac{(1+\alpha) \Gamma(k-1-\alpha)}{\Gamma(1-\alpha) \Gamma(k+1)} & \text { if } 0<\alpha<1,\end{cases}
$$

where $\Gamma$ is the gamma function. It is easily checked that the generating function

$$
F_{\alpha}(s, t)=1-\left(1-\mathrm{e}^{-t}+\mathrm{e}^{-t}(1-s)^{-\alpha}\right)^{-1 / \alpha}
$$

solves the forward Kolmogorov equation,

$$
\frac{\partial F_{\alpha}(s, t)}{\partial t}=\left(f_{\alpha}(s)-s\right) \frac{\partial F_{\alpha}(s, t)}{\partial s}
$$


thus, providing the generating function of $Z_{\alpha}(t)$. Note that $Z_{1}(t)$ is the well-known Yule process of binary splitting with

$$
F_{1}(s, t)=\frac{s \mathrm{e}^{-t}}{1-\left(1-\mathrm{e}^{-t}\right) s},
$$

implying that the distribution of $Z_{1}(t)$ is shifted geometric for all $t$.

To state our main result, we introduce a Markov branching process $Z_{0}(t)$ with infinite mean for the offspring number. Here the reproduction law is given by

$$
\mathrm{P}\left(v_{0}=k\right)=\frac{1}{k(k-1)}, \quad k=2,3, \ldots,
$$

corresponding to the generating function

$$
f_{0}(s)=s+(1-s) \log (1-s) .
$$

Thus, the part of (12) given for $0<\alpha<1$ is also valid for $\alpha=0$. The generating function of $Z_{0}(t)$ is

$$
F_{0}(s, t)=1-(1-s)^{\exp (-t)},
$$

which can be used to compute

$$
\mathrm{P}\left(Z_{0}(t)=1\right)=\mathrm{e}^{-t} .
$$

Theorem 2. Under the conditions of Theorem 1, the weak convergence of the processes over the time interval $0 \leq x<1$,

$$
(Z(t x, t) \mid Z(t)>0) \stackrel{\mathrm{D}}{\rightarrow} Z_{0}\left(-\frac{\beta}{1+\beta} \log (1-x)\right),
$$

holds in the Skorohod sense as $t$ tends to $\infty$.

From this result it is easy to recover (8) using (10) and (15). The limit process $Z_{0}(-\beta(1+$ $\left.\beta)^{-1} \log (1-x)\right)$ gives the following algorithm defining the genealogical tree:

- start with a single particle at time 0 which lives a random time $1-\tau_{0}$, where $\tau_{0}$ has density function (9);

- at time $1-\tau_{0}$ split the initial particle into a random number of daughter particles according to distribution (13);

- given $\tau_{0}$, let each daughter particle, independently of other particles, mimic the life of its mother, namely, let it live a time $\left(1-\tau_{1}\right) \tau_{0}$, where $\tau_{1}$ has density (9) and then split it using (13);

- given $\tau_{0}$ and $\tau_{1}$, let a granddaughter particle live a time $\left(1-\tau_{2}\right) \tau_{1} \tau_{0}$, where $\tau_{2}$ has density (9) and then split it using (13), and so on.

This should be compared with a similar algorithm describing the limit process in (11) for $0<\alpha \leq 1$, where the density (9) is replaced by the uniform density over $[0,1]$, and the offspring number distribution (13) is replaced by (12).

Figure 1 clearly indicates an interesting transformation of the limit law for the genealogical tree as the parameter $\alpha$ decreases from 1 to 0 , and then at $\alpha=0$ the new parameter $\beta$ goes from $\infty$ down to 0 . The $\alpha$-model has common branch length distribution and the value of 

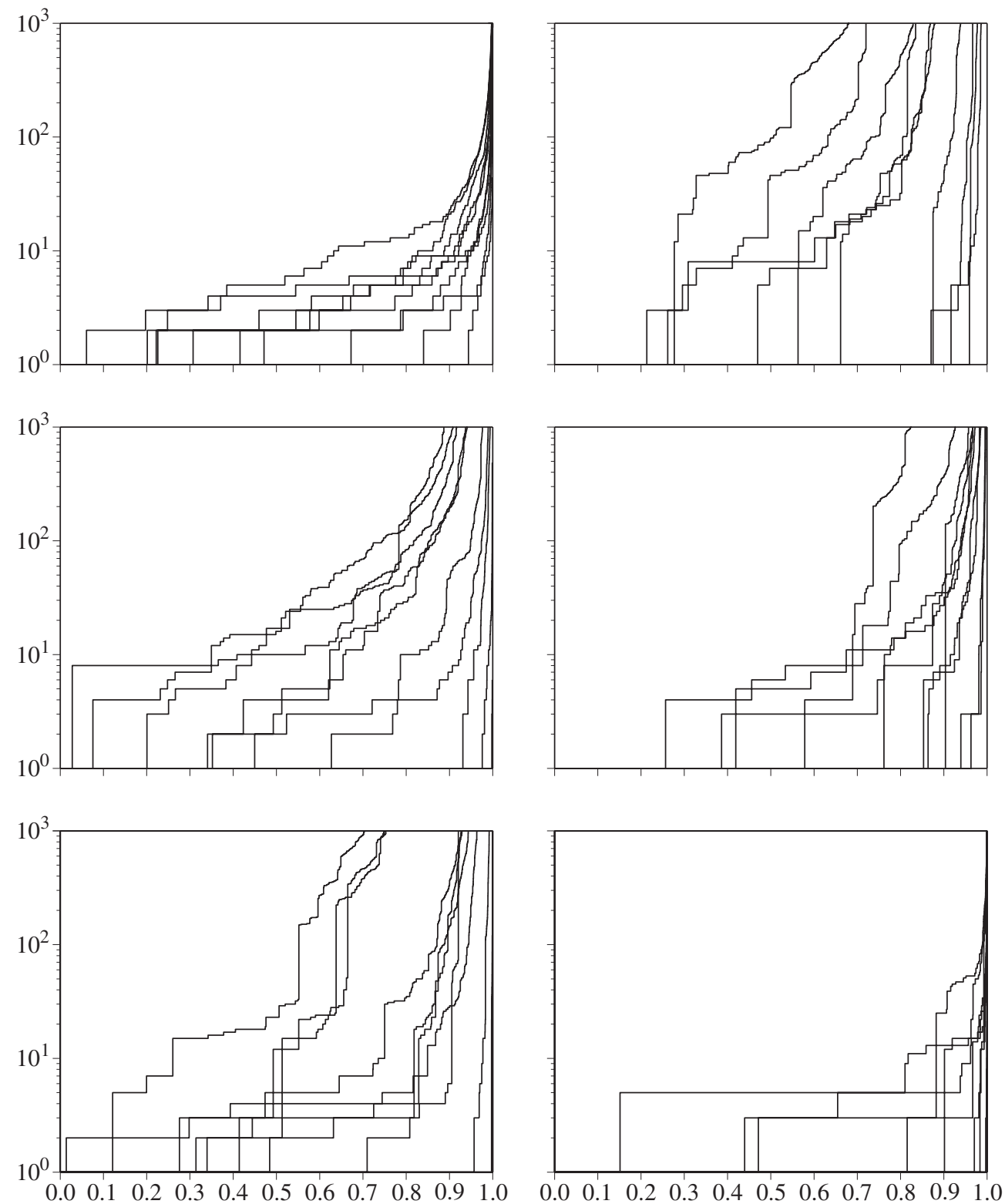

FIGURE 1: Six blocks with ten realizations each of the limit process for different parameter values. The three blocks on the left correspond to the values $\alpha=1$ (top row), $\alpha=0.3$ (middle row), and $\alpha=0.1$ (bottom row). The three blocks on the right correspond to the values $\beta=5$ (top row), $\beta=1$ (middle row), and $\beta=0.2$ (bottom row). For $\beta=0.2$, some trajectories are too close to 1 to be visible. The time to the most recent common ancestor is the horizontal distance from the right end of the time interval $[0,1)$ to the point where the trajectory leaves the state $1=10^{\circ}$.

parameter $\alpha$ determines the reproduction law, which smoothly changes from the deterministic splitting at $\alpha=1$ via (12) to distribution (13) with infinite mean at $\alpha=0$. It is easy to verify the stochastic domination property: if $0 \leq \alpha_{1} \leq \alpha_{2} \leq 1$ then $\mathrm{P}\left(v_{\alpha_{2}}>k\right) \leq \mathrm{P}\left(v_{\alpha_{1}}>k\right)$ for 
all $k$. This property is nicely illustrated by the left-hand side simulations of Figure 1: the lower the value of parameter $\alpha$, the faster the growth of the genealogical tree.

At $\alpha=0$, when the new parameter $\beta$ takes over the control, the dynamics of tree behavior drastically changes. As $\beta$ goes from larger to smaller values, it is the branch length (and not the reproduction) that undergoes transformation. Since the reproduction law (13) is common for all $\beta \in(0, \infty)$, we observe an opposite development of the tree growth: the closer $\beta$ is to 0 (and, therefore, the heavier the tail of the original reproduction law), the closer the splitting times are located to the observation time. Here we have another example of the phenomenon mentioned earlier concerning the critical value $\beta=1$. We observe a growth pattern (this time in terms of genealogical trees) which reaches its top and is then followed by a monotone decline.

Remark 1. This development in the tree growth depending on decreasing values of $\beta$ indicates that at the region $\beta=0$, the linear time scaling for the reduced process should be replaced by a nonlinear one in agreement with [11, Theorem 4(b)].

It was pointed out in [6] that the limit reproduction law for the critical reduced branching process with $\alpha \in(0,1]$ is related to the merging law of the $\Lambda$-coalescent with $\Lambda(\mathrm{d} x)=$ $(1-\alpha) x^{-\alpha} \mathrm{d} x$. Namely, if $Y_{n}$ is the size of the next merger given that there are currently $n$ branches then

$$
\mathrm{P}\left(Y_{n}=k\right)=\mathrm{P}\left(v_{\alpha}=k \mid v_{\alpha} \leq n\right) .
$$

Theorem 2 shows that there is a similar link between the reduced processes with $\alpha=0$ and the $\Lambda$-coalescent with uniform $\Lambda$, i.e. the Bolthausen-Sznitman coalescent (see [5]).

\section{Preliminary results}

If $f(s)>s$ for $0 \leq s<1$, the function

$$
\pi(s)=\int_{0}^{s} \frac{\mathrm{d} v}{f(v)-v}
$$

is obviously monotone and, consequently, $\rho(x)=\pi\left(1-\mathrm{e}^{-x}\right)$ as well. Let (1) hold with $\alpha=0$ and set $g(x)=L\left(\mathrm{e}^{x}\right)$, then condition (2) is equivalent to

$$
g(x) \sim x^{-\beta} L_{1}(x), \quad x \rightarrow \infty .
$$

Note that

$$
\begin{aligned}
\pi(s) & =\int_{1-s}^{1} \frac{\mathrm{d} t}{f(1-t)-1+t} \\
& =\int_{1-s}^{1} \frac{1}{L(1 / t)} \frac{\mathrm{d} t}{t} \\
& =\int_{0}^{-\ln (1-s)} \frac{\mathrm{d} w}{L\left(\mathrm{e}^{w}\right)} \\
& =\int_{0}^{-\ln (1-s)} \frac{\mathrm{d} w}{g(w)},
\end{aligned}
$$

therefore,

$$
\rho(x)=\int_{0}^{x} \frac{\mathrm{d} w}{g(w)},
$$


and it follows, from (16) and [1, Theorem 1.5.8], that

$$
\rho(x) \sim \frac{x^{\beta+1}}{(\beta+1) L_{1}(x)}, \quad x \rightarrow \infty .
$$

Lemma 1. $\operatorname{Set} q(t)=-\ln Q(t)$. Then

$$
\begin{gathered}
\rho(q(t))=t, \\
q^{\prime}(t)=g(q(t)) .
\end{gathered}
$$

Proof. The generating function $F(s, t)=\mathrm{E}\left[s^{Z(t)}\right]$ of the Markov branching process satisfies the backward Kolmogorov equation,

$$
\frac{\partial F(s, t)}{\partial t}=f(F(s, t))-F(s, t)
$$

with the boundary condition $F(s, 0)=s$. It follows that

$$
\pi(F(s, t))=\pi(s)+t .
$$

Setting $s=0$ yields the asserted equality (19),

$$
\pi(1-Q(t))=t,
$$

since $\pi(0)=0$ and $Q(t)=1-F(0, t)$. Furthermore, (21) and (22) give an important representation:

$$
1-F(s, t)=Q(\pi(s)+t) .
$$

After differentiating both sides of (19) we find that

$$
\rho^{\prime}(q(x))=\frac{1}{q^{\prime}(x)},
$$

which together with (17) implies (20).

Lemma 2. If (1) holds with $\alpha=0$ then (2) implies (3). On the other hand, if $\mathrm{E}[\mathrm{v}]=1$ then (3) implies (1) with $\alpha=0$ and (2) holds.

Proof. First note that

$$
1-f(s)=(1-s) \sum_{k=0}^{\infty} \mathrm{P}(v>k) s^{k},
$$

and, similarly, since $\mathrm{E}[v]=\sum_{k=0}^{\infty} \mathrm{P}(v>k)=1$,

$$
\frac{f(s)-s}{1-s}=(1-s) \sum_{k=0}^{\infty}\left(\sum_{i>k} \mathrm{P}(v>i)\right) s^{k} .
$$

Therefore, condition (1) with $\alpha=0$ is equivalent to

$$
\sum_{k=0}^{\infty}\left(\sum_{i>k} \mathrm{P}(v>i)\right) s^{k}=(1-s)^{-1} L\left(\frac{1}{1-s}\right) .
$$


According to [1, Corollary 1.7.3], the latter is equivalent to

$$
\sum_{i>k} \mathrm{P}(v>i) \sim L(k), \quad k \rightarrow \infty,
$$

or, in integral form,

$$
\int_{x}^{\infty} \mathrm{P}(v>y) \mathrm{d} y \sim L(x), \quad x \rightarrow \infty .
$$

Now given (2), we apply [1, Theorem 1.7.2b] to see that

$$
\int_{z}^{\infty} \mathrm{P}\left(v>\mathrm{e}^{y}\right) \mathrm{e}^{y} \mathrm{~d} y \sim z^{-\beta} L_{1}(z), \quad z \rightarrow \infty,
$$

entails

$$
\mathrm{P}\left(v>\mathrm{e}^{z}\right) \mathrm{e}^{z} \sim \beta z^{-1-\beta} L_{1}(z), \quad z \rightarrow \infty,
$$

which is (3). To prove the assertion in the opposite direction, we should apply [1, Theorem 1.5.11].

Lemma 3. Under the conditions of Theorem 1, as $x \rightarrow \infty$,

$$
\begin{aligned}
g^{\prime}(x) & \sim-\frac{\beta g(x)}{x}, \\
q^{\prime \prime}(x) & \sim \frac{\beta q^{\prime}(x)^{2}}{q(x)}, \\
\rho^{\prime \prime}(q(x)) & \sim \frac{\beta}{q^{\prime}(x) q(x)} .
\end{aligned}
$$

If we define $c(t)$ by

$$
\frac{1}{c(t)}=q\left(\frac{1}{g(q(t))}\right)
$$

then it will satisfy (5).

Proof. In the critical case we have

$$
\begin{aligned}
1-f^{\prime}(s) & =(1-s) \sum_{k=0}^{\infty}\left(\sum_{i=k+2}^{\infty} i \mathrm{P}(v=i)\right) s^{k} \\
& =(1-s) \sum_{k=0}^{\infty} \mathrm{E}\left[v \mathbf{1}_{\{v \geq k+2\}}\right] s^{k} .
\end{aligned}
$$

On the other hand, owing to (25), we have

$$
\begin{aligned}
f(s)-s & =(1-s)^{2} \sum_{k=0}^{\infty}\left(\sum_{i>k} \mathrm{P}(v>i)\right) s^{k} \\
& =(1-s)^{2} \sum_{k=0}^{\infty} \mathrm{E}\left[(v-k-1) \mathbf{1}_{\{v \geq k+2\}}\right] s^{k} .
\end{aligned}
$$


These two relations together with $L\left((1-s)^{-1}\right)=(f(s)-s) /(1-s)$ yield

$$
\begin{aligned}
\frac{1}{(1-s)^{2}} L^{\prime}\left(\frac{1}{1-s}\right) & =\frac{f^{\prime}(s)-1}{1-s}+\frac{f(s)-s}{(1-s)^{2}} \\
& =-\sum_{k=0}^{\infty}\left(\mathrm{E}\left[v \mathbf{1}_{\{v \geq k+2\}}\right]-\mathrm{E}\left[(v-k-1) \mathbf{1}_{\{v \geq k+2\}}\right]\right) s^{k} \\
& =-\sum_{k=0}^{\infty}(k+1) \mathrm{P}(v>k+1) s^{k}
\end{aligned}
$$

thus, owing to (3), as $s \rightarrow 1$,

$$
L^{\prime}\left(\frac{1}{1-s}\right) \sim-\beta(1-s)^{2} \sum_{k=0}^{\infty}(\ln k)^{-1-\beta} L_{1}(\ln k) s^{k} .
$$

Since, owing to [1, Proposition 1.5.8],

$$
\sum_{k=0}^{n}(\ln k)^{-1-\beta} L_{1}(\ln k) \sim n(\ln n)^{-1-\beta} L_{1}(\ln n),
$$

we derive from the previous relation, applying [1, Corollary 1.7.3],

$$
\frac{1}{1-s} L^{\prime}\left(\frac{1}{1-s}\right) \sim-\beta|\ln (1-s)|^{-1-\beta} L_{1}(-\ln (1-s)) \text {. }
$$

Now (26) follows from the relations $g^{\prime}(x)=\mathrm{e}^{x} L^{\prime}\left(\mathrm{e}^{x}\right)$ and (2).

To derive (27), it is enough to observe that $q^{\prime \prime}(x)=g^{\prime}(q(x)) q^{\prime}(x)$ and use (26). From (24) we obtain $\rho^{\prime \prime}(q(x))=-q^{\prime \prime}(x) / q^{\prime}(x)^{3}$. This and (27) give (28). The last assertion of the lemma is a simple consequence of the basic properties of regular varying functions.

\section{Proofs of Theorems 1 and 2}

The asymptotics (4) of $Q(t)$ stated in Theorem 1 follow from (18) and (19) in view of [1, Theorem 1.5.12].

We prove (6) and Theorem 2 after deriving an expression for the generating function of the reduced process

$$
\tilde{F}(s ; u, t)=\mathrm{E}\left[s^{Z(u, t)}\right]
$$

in terms of $F(s, t)$. The survival probability at time $t$ for a branching process starting from a single particle at time $u$ is equal to $Q(t-u)$. Since the total number of particles alive at time $u$ is described by $F(s, u)$, we can write

$$
\tilde{F}(s ; u, t)=F(1-Q(t-u)+Q(t-u) s, u) .
$$

Combining this with the obvious relation

$$
\tilde{F}(s ; u, t)=1-Q(t)+\mathrm{E}\left[s^{Z(u, t)} \mid Z(t)>0\right] Q(t),
$$


we deduce that

$$
\begin{aligned}
\mathrm{E}\left[s^{Z(u, t)} \mid Z(t)>0\right] & =\frac{\tilde{F}(s ; u, t)-1+Q(t)}{Q(t)} \\
& =1-\frac{1-F(1-Q(t-u)+Q(t-u) s, u)}{Q(t)} .
\end{aligned}
$$

In view of (23) it follows that

$$
\begin{aligned}
\mathrm{E}\left[s^{Z(u, t)} \mid Z(t)>0\right] & =1-\frac{Q(\pi(1-Q(t-u)(1-s))+u)}{Q(t)} \\
& =1-\exp (q(t)-q(t+\Delta(s, t-u))),
\end{aligned}
$$

where

$$
\Delta(s, t)=\rho(q(t)-\ln (1-s))-t .
$$

Using (30) with $u=t$, we obtain

$$
-\ln \left(1-\mathrm{E}\left[s^{Z(t)} \mid Z(t)>0\right]\right)=q(\rho(-\ln (1-s))+t)-q(t) .
$$

To prove (6), we study (31) with $s=1-\mathrm{e}^{-x / c(t)}$, where $x$ is a fixed positive number and $c(t)$ is defined by (29). According to Lemma 3 and (18),

$$
\rho\left(\frac{x}{c(t)}\right) \sim \frac{x^{1+\beta}}{q^{\prime}(t)}, \quad t \rightarrow \infty,
$$

and, therefore, by a Taylor expansion around $t$,

$$
\begin{aligned}
-\ln \left(1-\mathrm{E}\left[\left(1-\mathrm{e}^{-x / c(t)}\right)^{Z(t)} \mid Z(t)>0\right]\right) & =q\left(\rho\left(\frac{x}{c(t)}\right)+t\right)-q(t) \\
& =\rho\left(\frac{x}{c(t)}\right) q^{\prime}(t)+\rho\left(\frac{x}{c(t)}\right)^{2} O\left(q^{\prime \prime}(t)\right) \\
& \rightarrow x^{1+\beta} .
\end{aligned}
$$

Thus,

$$
\mathrm{E}\left[\left(1-\mathrm{e}^{-x / c(t)}\right)^{Z(t)} \mid Z(t)>0\right] \rightarrow 1-\exp \left(-x^{1+\beta}\right)
$$

and, by the arguments of [2] and [7] or [4], this implies that

$$
\mathrm{P}(\ln Z(t) \leq x c(t) \mid Z(t)>0) \rightarrow 1-\exp \left(-x^{1+\beta}\right),
$$

which completes our proof of Theorem 1 .

The proposed limit process $R(x)=Z_{0}\left(-\beta(1+\beta)^{-1} \log (1-x)\right)$ in Theorem 2 is a timeinhomogeneous Markov branching process, and from (14) we find that

$$
\mathrm{E}\left[s^{R(y)} \mid R(x)=1\right]=1-(1-s)^{((1-y) /(1-x))^{\beta /(1+\beta)}}, \quad 0 \leq x \leq y<1 .
$$

This yields

$$
\mathrm{P}(R(y)=1 \mid R(x)=1)=\left(\frac{1-y}{1-x}\right)^{\beta /(1+\beta)} .
$$


By similar arguments that led to (30), we have

$$
\mathrm{E}\left[s^{Z(v, t)} \mid Z(u, t)=1, Z(t)>0\right]=1-\exp (q(t-u)-q(\Delta(s, t-v)+t-u))
$$

for $0 \leq u \leq v<t$. In order to prove convergence of finite-dimensional distributions it suffices to show that

$$
q(\Delta(s, x t)+y t)-q(y t) \rightarrow-\left(\log (1-s)^{(x / y)^{\beta /(1+\beta)}}\right), \quad 0<x \leq y \leq 1 .
$$

We do this in two steps. Firstly, we find the asymptotics of $\Delta(s, t)$ by a Taylor expansion of the function $\rho$ around $q$ :

$$
\begin{aligned}
\Delta(s, t) & =\rho(q(t)-\ln (1-s))-\rho(q(t)) \\
& =-\ln (1-s) \rho^{\prime}(q(t))+O\left(\rho^{\prime \prime}(q(t))\right) \\
& \sim-\frac{\ln (1-s)}{q^{\prime}(t)} .
\end{aligned}
$$

Secondly, we do another Taylor expansion, this time of the function $q$ around $y t$ :

$$
\begin{aligned}
q(y t+\Delta(s, x t))-q(y t) & =-\ln (1-s) \frac{q^{\prime}(y t)}{q^{\prime}(x t)}+\left(q^{\prime}(t)\right)^{-2} O\left(q^{\prime \prime}(t)\right) \\
& \rightarrow-\ln (1-s)\left(\frac{x}{y}\right)^{\beta /(1+\beta)},
\end{aligned}
$$

according to Lemma 3. This completes the proof of the convergence of finite-dimensional distributions in Theorem 2.

To show the convergence in the Skorohod sense, we can now copy the proof in [3] almost verbatim. The only difference is that we have (32) with $0<\beta /(1+\beta)<1$, whereas they, in our notation, have the expression $(1-y) /(1-x)$ for the corresponding probability. The difference in the exponent has no consequence for the proof.

\section{Acknowledgement}

We thank V. Wachtel for stimulating discussions of his recent paper [4].

\section{References}

[1] Bingham, N. H., Goldie, C. M. and Teugels, J. L. (1987). Regular Variation. Cambridge University Press.

[2] Darling, D. A. (1970). The Galton-Watson process with infinite mean. J. Appl. Prob. 7, 455-456.

[3] Fleischmann, K. and Siegmund-Schultze, R. (1977). The structure of reduced critical Galton-Watson processes. Math. Nachr. 79, 233-241.

[4] Nagaev, S. V. and Wachtel, V. (2007). The critical Galton-Watson process without further power moments. J. Appl. Prob. 44, 753-769.

[5] Pitman, J. (1999). Coalescents with multiple collisions. Ann. Prob. 27, 1870-1902.

[6] Sagitov, S. (1999). The general coalescent with asynchronous mergers of ancestral lines. J. Appl. Prob. 36, 1116-1125.

[7] Seneta, E. (1973). The simple branching process with infinite mean. I. J. Appl. Prob. 10, 206-212.

[8] SLACK, R. S. (1968). A branching process with mean one and possibly infinite variance. Z. Wahrscheinlichkeitsth. 9, 139-145.

[9] Yakymiv, A. L. (1980). Reduced branching processes. Theory Prob. Appl. 25, 584-588.

[10] Zolotarev, V. M. (1957). More exact statements of several theorems in the theory of branching processes. Theory Prob. Appl. 2, 245-253.

[11] Zubkov, A. M. (1975). Limit distributions of the distance to the nearest common ancestor. Theory Prob. Appl. 20, 602-612. 